\title{
Editorial: Welcoming Chris Leighton to Physical Review Materials
}

As we proudly publish the first issue of Physical Review Materials (PR Materials), it is my distinct pleasure and honor to introduce you to its founding Lead Editor, Dr. Chris Leighton. I could not be more pleased that Chris agreed to take on this challenging task, and I am certain that PR Materials, and the full family of APS journals as well, will benefit greatly from his leadership, energy, wisdom, and broad vision.

Chris is a Distinguished McKnight University Professor of Chemical Engineering and Materials Science and a graduate faculty member in Physics at the University of Minnesota. Following Ph.D. studies in Condensed Matter Physics at the University of Durham in the UK, he pursued post-doctoral research at the University of California San Diego before joining the University of Minnesota in 2001. His research deals with the electronic and magnetic properties of a wide range of novel materials, including complex oxides, oxide heterostructures, metallic spintronics, complex alloys, organic conductors, and photovoltaics. He is a Fellow of the APS, was selected in 2012 as an APS Outstanding Referee, and currently serves as Chair of the Topical Group on Magnetism and its Applications (GMAG). His honors also include a 2014 Cozzarelli Prize in Engineering and Applied Sciences from the Proceedings of the National Academy of Sciences.

Please join me in welcoming Chris as he takes on the editorial leadership of the newest member of the APS family of journals. After reading his Editorial you will surely agree with me that we are in really good hands!

Pierre Meystre Editor in Chief

Published 19 June 2017

DOI: 10.1103/PhysRevMaterials.1.010001 